Grupo de Brauer e o teorema de Merkurjev-Suslin Gilberto Luiz Angelice de Camargo 
SERVIÇO DE PÓS-GRADUAÇÃO DO ICMC-USP

Data de Depósito:

Assinatura:

\title{
Grupo de Brauer e o teorema de Merkurjev-Suslin
}

\author{
Gilberto Luiz Angelice de Camargo
}

Orientador: Prof. Dr. Eduardo Tengan

Dissertação apresentada ao Instituto de Ciências Matemáticas e de Computação - ICMC-USP, como parte dos requisitos para obtenção do título de Mestre em Ciências - Matemática. EXEMPLAR DE DEFESA.

USP - São Carlos

Março de 2013 
Ficha catalográfica elaborada pela Biblioteca Prof. Achille Bassi e Seção Técnica de Informática, ICMC/USP, com os dados fornecidos pelo(a) autor(a)

Angelice de Camargo, Gilberto Luiz
A172gupo de Brauer e o teorema de Merkurjev-Suslin
/ Gilberto Luiz Angelice de Camargo; orientador
Eduardo Tengan. -- São Carlos, 2013.
$27 \mathrm{p}$.
Dissertação (Mestrado - Programa de Pós-Graduação
em Matemática) -- Instituto de Ciências Matemáticas
e de Computação, Universidade de São Paulo, 2013.
1. Grupo de Brauer. 2. Teorema de Merkurjev-
Suslin. 3. Álgebra de Quatérnios. I. Tengan,
Eduardo, orient. II. Título.


Um sonho só é impossivel se você não acreditar o suficiente 



\section{Agradecimentos}

Primeiramente gostaria de agradecer aos meus orientadores, Daniel e Eduardo, por todo conhecimento adquirido, pela experiência acadêmica. De modo especial, agradeço ao Eduardo por todos os conselhos acadêmicos, pessoais e profissionais, também por toda ajuda e empenho que me ofereceu durante toda essa caminhada, me deixando um grande exemplo de professor e amigo. Muito obrigado!

À toda minha família, pela força e apoio que sempre me deram em cada momento, tenha sido ele fácil ou difícil. Um agradecimento muito especial à minha mãe Gislene, ao meu tio Luiz Carlos e minha irmã Lais, sem vocês jamais seria quem eu sou hoje e jamais chegaria até onde cheguei.

Agradeço também a todos os meus amigos pois sem vocês eu não seria ninguém. Aos meus amigos de Barra Bonita e São Carlos pelos momentos felizes que me proporcionaram. Em especial eu agradeço a Rafa, Martha, André, Rafael, Amanda, Mariana, Vitor, Yasmin, Tiago, Murilo, Sender, Igor, Cássio, Tiago, Neto por cada risada e momentos felizes que me proporcionaram e em especial ao Allan que me acompanhou por grande parte desta jornada e se foi tão cedo deixando uma imensa saudades. A vocês um imenso obrigado.

Por fim, agradeço à FAPESP pelo apoio financeiro. 



\section{Resumo}

Neste trabalho mostramos o importante teorema de MerkurjevSuslin para o caso de 2-torção, seguindo o artigo [Mer06], que afirma que, para qualquer corpo $F$ de característica diferente de 2, a 2-torção ${ }_{2} B r(F)$ do grupo de Brauer de $F$ é gerada pelas classes de álgebras de quatérnions. 

In this work we show the important theorem of MerkurjevSuslin for 2-torsion, following the paper [Mer06], which states that for any field $F$ of characteristic not 2 the 2-torsion ${ }_{2} \operatorname{Br}(F)$ of the Brauer Group of $F$ is generated by the quaternion algebra classes. 

Introdução

1 Álgebras Centrais Simples $\quad 1$

1.1 Notações . . . . . . . . . . . . . . . . . . . . . . . . . . . . . . . . 1

1.2 Álgebras Centrais Simples . . . . . . . . . . . . . . . . 2

2 Grupo de Brauer $\quad 7$

2.1 Descenso de Galois . . . . . . . . . . . . . . . . . . . . . 7

2.2 Grupo de Brauer . . . . . . . . . . . . . . . . . . . 11

3 Teoria $K$ de Milnor $\quad 13$

3.1 Definições e Propriedades Básicas . . . . . . . . . . . . . . . . . . . . . 13

3.2 Morfismos Residual e de Especialização . . . . . . . . . . . . . . . . . . 14

3.3 Norma . . . . . . . . . . . . . . . . . . 15

4 Geometria das Curvas Cônicas $\quad 17$

4.1 Quatérnios ............................ 17

4.2 Norma e Traço Reduzidos . . . . . . . . . . . . . . . . . . 18

4.3 Cônicas e Quatérnios . . . . . . . . . . . . . . . . . . . 19

5 O Teorema de Merkurjev-Suslin $\quad 21$

5.1 Símbolo de Galois . . . . . . . . . . . . . . . . . . . . 21

5.2 Demonstração do Teorema de Merkurjev-Suslin . . . . . . . . . . . . . 22

$\begin{array}{ll}\text { Referências Bibliográficas } & 27\end{array}$ 

O teorema de Merkurjev-Suslin [MS82] afirma que, para todo corpo $F \operatorname{com} \operatorname{char}(F) \neq$ $n$, temos um isomorfismo

$$
h_{F}: \frac{K_{2} F}{n K_{2} F} \rightarrow H^{2}\left(G_{F}, \mu_{n} \otimes \mu_{n}\right)
$$

- Em termos de álgebras centrais simples, o isomorfismo acima pode ser interpretado da seguinte forma: se $F$ contém todas as raízes $n$-ésimas da unidade, então a $n$-torção do grupo de Brauer ${ }_{n} B r(F)$ é gerada por álgebras cíclicas.

Neste trabalho provaremos o caso particular em que $n=2$. Para isto, seguiremos o artigo [Mer06] de A. Merkurjev, onde ele dá uma prova que simplifica a prova original do artigo [MS82].

Nos capítulos de 1 a 4 apresentamos alguns dos pré-requisitos para a prova do teorema de Merkurjev-Suslin, que é dada no capítulo 5. No capítulo 1 discutimos algumas propriedades elementares de álgebras centrais simples. No capítulo 2 definimos o grupo de Brauer e o caracterizamos como um grupo de cohomologia. No capítulo 3 listamos algumas propriedades dos grupos $K$ de Milnor. No capítulo 4 discutimos álgebras de quatérnios e sua relação com cônicas projetivas planas. E por fim no capítulo 5 provamos o teorema de Merkurjev-Suslin propriamente dito. 



\begin{tabular}{l|l|}
\hline Capítulo \\
\hline 7 \\
\hline
\end{tabular}

\section{Álgebras Centrais Simples}

Neste capítulo estudaremos as álgebras centrais simples, onde veremos o teorema de Wedderburn e também a classificação de todas as álgebras centrais simples com centro em um corpo $K$, temos como resultado principal o corolário 1.10.

\subsection{Notações}

Assumiremos no texto a seguir algumas notações e convenções:

- Todas as álgebras serão associativas

- quando não definido assumiremos que $K$ é um corpo.

- todos os módulos e ideais serão à esquerda.

- $Z(A)$ será o centro do anel $A$.

- uma álgebra de divisão $D$ sobre um corpo $K$ é uma álgebra de divisão de dimensão finita sobre $K$ e tal que $Z(D)=K$.

- Se $V$ é um espaço vetorial então $V^{*}$ denotará o espaço dual de $V$ 


\section{2 Álgebras Centrais Simples}

Definição 1.1. Dizemos que uma $K$-álgebra associativa $A$ de dimensão finita é uma álgebra central simples (usaremos a notação CSA) se A é um anel simples com centro $K$.

Exemplo 1.2. Se $D$ é uma álgebra de divisão sobre $K$, então o anel $M_{n}(D)$ das matrizes $n \times n$ sobre $D$ é simples para todo $n \geq 1$.

Demonstração. Verificar isso é um simples exercício de matrizes, para isso devemos provar que todo ideal bilateral $\langle M\rangle$ em $M_{n}(D)$ gerado por uma matriz $M$ não nula é $M_{n}(D)$. Considere $E_{i j}$ a matriz que tem 1 na $j$-ésima entrada da $i$-ésima linha e zero nas demais coordenadas. Assim cada elemento de $M_{n}(D)$ é uma combinação $D$-linear dos $E_{i j}$, assim basta provarmos que $E_{i j} \in\langle M\rangle$ para todo $i, j$. Observe que $E_{k i} E_{i j} E_{j l}=E_{k l}$ logo precisamos provar que $E_{i j} \in\langle M\rangle$ para algum $i, j$. Agora escolha um $i, j$ tal que a $j$-ésima entrada da $i$-ésima columa da matriz $M$ seja um $m$ diferente de zero. Então temos que $m^{-1} E_{i i} M E_{j j}=E_{i j}$, e temos o resultado.

Repare que é fácil ver que o centro do anel das matrizes contém somente múltiplos escalares da unidade, assim temos que $M_{n}(D)$ é uma álgebra central simples.

Lema 1.3. (Schur) Seja $M$ um módulo simples sobre uma $K$-álgebra $A$. Então $\operatorname{End}_{A}(M)$ é uma álgebra de divisão.

Demonstração. Seja $\phi \in \operatorname{End}_{A}(M), \phi \neq 0, \operatorname{logo} \operatorname{Ker} \phi$ é um submódulo de $M$ e como $\phi \neq 0$ temos que $\operatorname{Ker} \phi \neq M$, portanto $\operatorname{Ker} \phi=\{0\}$, e $\operatorname{Im} \phi$ também é um submódulo de $M$ onde $\operatorname{Im} \phi \neq\{0\}$, com isso $\operatorname{Im} \phi=M$ e assim $\phi$ é um isomorfismo.

Podemos agora definir em $M$ uma estrutura de módulo sobre $D=\operatorname{End}_{A}(M)$, onde $\phi \cdot m=\phi(m)$. Assim vamos considerar agora o anel dos endomorfismos $\operatorname{End}_{D}(M)$. Definiremos o seguinte morfismo de anéis

$$
\begin{aligned}
\lambda_{M}: A & \rightarrow \operatorname{End}_{D}(M) \\
a & \mapsto(x \mapsto a x)
\end{aligned}
$$

Temos que $\lambda(a)$ é realmente um $D$-endomorfismo, para isso seja $\phi \in D$, temos que $\phi \cdot a x=\phi(a x)=a \phi(x)=a \phi \cdot x$ para todo $x \in M$.

Lema 1.4 (Rieffel). Seja $L$ um ideal à esquerda não nulo de uma K-álgebra simples $A$ e $D=\operatorname{End}_{A}(L)$. Então o mapa $\lambda_{L}: A \rightarrow \operatorname{End}_{D}(L)$ como definido acima é um isomorfismo. 
Note que um ideal à esquerda nada mais é do que um submódulo do $A$-módulo $A$.

Demonstração. Como $\lambda_{L} \neq 0$, temos que o núcleo é um ideal bilateral próprio de A. Mas como $A$ é simples segue que $\operatorname{Ker} \lambda_{L}=\{0\}$, com isso $\lambda_{L}$ é injetiva. Para a sobrejetividade mostraremos primeiro que $\lambda_{L}(L)$ é um ideal à esquerda de $\operatorname{End}_{D}(L)$. Para cada $x \in L, R_{x}: L \rightarrow L$ dada por $R_{x}(l)=l x$ é um $A$-endomorfismo de $L$, isto é um elemento de $D$. Seja $\phi \in \operatorname{End}_{D}(L) \operatorname{logo}$

$$
\phi \cdot \lambda_{L}(l)(x)=\phi(l x)=\phi\left(R_{x}(l)\right)=R_{x}(\phi(l))=\phi(l) x=\lambda_{L}(\phi(l))(x)
$$

para todo $x \in L$, assim $\phi \cdot \lambda_{L}(l)=\lambda_{L}(\phi(l)) \in \lambda_{L}(L)$, portanto $\lambda_{L}(L)$ é um ideal à esquerda de $\operatorname{End}_{D}(L)$. Observe agora que $L A$ é um ideal bilateral de $A$ não nulo, segue que $L A=A$, em particular temos que $1 \in L A$ e assim $1=\sum l_{i} a_{i}$, com isso

$$
\begin{aligned}
\phi & =\phi \cdot 1_{D}=\phi \lambda_{L}(1)=\phi \lambda_{L}\left(\sum l_{i} a_{i}\right) \\
& =\phi\left(\sum \lambda_{L}\left(l_{i}\right) \lambda_{L}\left(a_{i}\right)\right)=\sum \phi \lambda_{L}\left(l_{i}\right) \lambda_{L}\left(a_{i}\right)
\end{aligned}
$$

Como $\lambda_{L}(L)$ é um ideal à esquerda temos que $\phi \lambda_{L}\left(l_{i}\right) \in \lambda_{L}(L)$. Seja $r_{i}=\phi\left(l_{i}\right)$ então $\phi \lambda_{L}\left(l_{i}\right)=\lambda_{L}\left(r_{i}\right) \in \lambda_{L}(L)$ e segue de (1.2.1) que

$$
\phi=\sum \phi \lambda_{L}\left(l_{i}\right) \lambda_{L}\left(a_{i}\right)=\sum \lambda_{L}\left(r_{i}\right) \lambda_{L}\left(a_{i}\right)=\sum \lambda_{L}\left(\phi\left(l_{i}\right) \cdot a_{i}\right)=\lambda_{L}\left(\sum \phi\left(l_{i}\right) \cdot a_{i}\right)
$$

o que implica que $\phi \in \lambda_{L}(A)$ e resulta que $\lambda_{L}$ é sobrejetivo.

Teorema 1.5. (Wedderburn) Se A é uma álgebra simples sobre $K$, existe um inteiro $n \geq 1$ e uma álgebra de divisão $D \supseteq K$ tal que $A$ é isomorfo ao anel $M_{n}(D)$. Além disso a álgebra de divisão é unicamente determinada por A a menos de isomorfismo.

Demonstração. Como $A$ tem dimensão finita, uma cadeia descendente de ideais à esquerda se estabiliza ou seja $A$ é artiniano. Logo seja $L$ um ideal minimal não nulo à esquerda, assim $L$ é um $A$-módulo simples, pelo lema de Schur $D=\operatorname{End}_{A}(L)$ é uma álgebra de divisão.

Pelo lema de Rieffel temos um isomorfismo $A \cong \operatorname{End}_{D}(L)$, como $D$ é uma álgebra de divisão temos que $\operatorname{End}_{D}(L) \cong M_{n}(D)$ onde $n$ é a dimensão de $L$ sobre $D$.

Para provar a unicidade, assuma que $D$ e $D^{\prime}$ sejam duas álgebras de divisão com $A \cong M_{n}(D) \cong M_{m}\left(D^{\prime}\right)$. Logo temos que o ideal minimal à esquerda satisfaz $D^{n} \cong$ $L \cong D^{\prime m}$ de onde seguem os seguintes isomorfismos

$$
D \cong \operatorname{End}_{A}\left(D^{n}\right) \cong \operatorname{End}_{A}(L) \cong \operatorname{End}_{A}\left(D^{\prime m}\right) \cong D^{\prime}
$$


Lema 1.6. Seja $K$ um corpo e sejam $A$ e $B$ duas $K$-álgebras de dimensão finita.

1. $Z\left(A \otimes_{K} B\right)=Z(A) \otimes_{K} Z(B)$.

2. Se $A$ e $B$ são anéis simples com $Z(A)=K$ então $A \otimes_{K} B$ tambem é simples $e$ $Z\left(A \otimes_{K} B\right)=Z(B)$

Demonstração. $\quad$ 1. $Z(A) \otimes_{K} Z(B) \subset Z\left(A \otimes_{K} B\right)$ segue trivialmente das definições do produto tensorial.

Vamos então provar $Z(A) \otimes Z(B) \supset Z(A \otimes B)$. Seja $\omega_{1}, \ldots, \omega_{n}$ uma base de $B$ sobre $K \log O$

$$
A \otimes_{K} B=A \otimes_{K}\left(\bigoplus_{1 \leq i \leq n} K \omega_{i}\right)=\bigoplus_{1 \leq i \leq n}\left(A \otimes_{K} K \omega_{i}\right)
$$

como $K$-espaço vetorial. Assim se $z \in A \otimes_{K} B$, podemos escrever $z=\sum_{1 \leq i \leq n} a_{i} \otimes$ $\omega_{i}$, se $z \in Z\left(A \otimes_{K} B\right)$ então

$$
\begin{aligned}
\left(a \otimes_{K} 1\right) \cdot z & =z \cdot\left(a \otimes_{K} 1\right) \\
& \Leftrightarrow a a_{1} \otimes_{K} \omega_{1}+\cdots+a a_{n} \otimes_{K} \omega_{n}=a_{1} a \otimes_{K} \omega_{1}+\cdots+a_{n} a \otimes_{K} \omega_{n}
\end{aligned}
$$

logo pela unicidade da representação $a a_{i}=a_{i} a$, para todo $a \in A$. Com isso $a_{i} \in Z(A)$ e portanto $Z(A) \otimes B \supset Z(A \otimes B)$. Por um argumento análogo vale $Z(A) \otimes_{K} Z(B) \supset Z\left(A \otimes_{K} B\right)$.

2. Seja $I$ um ideal bilateral de $A \otimes_{K} B$. Suponha que exista um "termo simples" não nulo $a \otimes_{K} b \in I$. Como $A$ é simples o ideal bilateral gerado por $a \neq 0$ é igual a $A, \operatorname{logo}$ existem $a_{i}$ e $a_{i}^{\prime}$ tais que

$$
\sum_{i=1}^{n} a_{i} a a_{i}^{\prime}=1
$$

Assim $1 \otimes_{K} b=\sum_{i=1}^{n}\left(a_{i} \otimes_{K} 1\right) \cdot\left(a \otimes_{K} b\right) \cdot\left(a_{i}^{\prime} \otimes_{K} 1\right)$ implicando que $1 \otimes_{K} b \in I$. Aplicando o mesmo processo para $B$ obtemos que $1 \otimes 1 \in I$, que implica $I=A \otimes B$. Agora seja $x=a_{1} \otimes b_{1}+\cdots+a_{n} \otimes b_{n} \in I$, um elemento com o menor $n$, podemos assumir que todos os $b_{i}^{\prime} s$ e $a_{i}^{\prime} s$ são linearmente independentes sobre $K$, pois caso contrário poderíamos encurtar a sequência.

Sem perda de generalidade vamos supor $a_{1}=1$.

Podemos supor $n>1$ pois o caso $n=1$ segue acima, logo temos que $a_{1} \neq a_{2}$ e $a_{2} \notin K$ (pois caso contrário $a_{1}$ e $a_{2}$ seriam linearmente dependentes). Como 
$Z(A)=K$ então existe $a \in A$ tal que $a a_{2} \neq a_{2} a$. Vamos considerar agora o elemento

$$
(a \otimes 1) x-x(a \otimes 1)=\left(a a_{2}-a_{2} a\right) \otimes b_{2}+\cdots+\left(a a_{n}-a_{n} a\right) \otimes b_{n}
$$

Como os $b_{i}^{\prime} s$ são linearmente independentes e $a a_{2} \neq a_{2} a$ temos que o elemento acima é não nulo o que contradiz a minimalidade de $n$, o que implica $n=1$.

Teorema 1.7. A é um anel simples com centro $K$ se e somente se $A \cong M_{n}(D)$ onde $D$ é uma álgebra de divisão com centro $K$.

Demonstração. $(\Rightarrow)$ Segue diretamente do Teorema de Wedderburn.

$(\Leftarrow)$ Através de cálculos simples com matrizes conseguimos mostrar que $M_{n}(D)$ é um anel simples com centro $K$.

Vamos mostrar agora que não existe uma álgebra de divisão não trivial $D$ sobre um corpo $\Omega$ algebricamente fechado. De fato temos que $\operatorname{dim}_{\Omega} D<\infty$, logo para todo $a \in D$ temos $\Omega(a)$ é um subcorpo com dimensão finita sobre $\Omega$ e portanto $\Omega(a)=\Omega$ $\Longrightarrow a \in \Omega$. O que prova que $D=\Omega$.

Seja $\Omega$ um corpo algebricamente fechado, e $A_{\Omega}=A \otimes \Omega$.

Teorema 1.8. A é um anel simples com centro $K$ se e somente se $A_{\Omega}$ é isomorfo ao anel das matrizes $M_{d}(\Omega)$ para algum $d$.

Demonstração. $(\Rightarrow)$ Como $A$ é um anel simples com centro $K$, temos pelo lema anterior que $A_{\Omega}$ é um anel simples com centro $\Omega$, assim pelo teorema de Wedderburn temos que $A_{\Omega}$ é isomorfo ao anel das matrizes $M_{d}(D)$ onde $D$ é uma álgebra de divisão que contém $\Omega$, porém como visto na observação acima temos que $D=\Omega$.

$(\Leftarrow)$ Observe primeiro que $\Omega$ é uma $K$-álgebra livre sobre $K$, assim $\Omega$ é fielmente plano sobre $K$, relembramos que isso quer dizer que $-\otimes \Omega$ é um funtor exato tal que $M \otimes \Omega=0 \Longleftrightarrow M=0$ para todo $K$-módulo $M$. Em particular $-\otimes \Omega$ preserva mapas injetivos.

Assim se existir um ideal bilateral não trivial $I$ de $A$ teremos que $I \otimes \Omega$ será um ideal bilateral de $A \otimes \Omega \cong M_{n}(\Omega)$ o que contradiz o fato de $M_{n}(\Omega)$ ser simples. Logo $A$ é simples. Por outro lado temos pelo lema anterior que $Z(A) \otimes_{K} \Omega=Z(A \otimes \Omega)=$ $Z\left(M_{n}(\Omega)\right)=\Omega$, assim $Z(A) \otimes_{K} \Omega=K \otimes_{K} \Omega$ como $\Omega$ é fielmente plano temos que $Z(A)=K$ como queríamos. 
Teorema 1.9. A é um anel simples de centro $K$ se e somente se o mapa canônico $\phi: A \otimes A^{o p} \rightarrow \operatorname{End}_{k-m o d}(A)$ é um isomorfismo. O mapa canônico é dado por $a \otimes b \mapsto f$ onde $f(x)=a x b$.

Demonstração. $(\Rightarrow)$ Observe primeiro que pelo lema anterior temos que $A \otimes_{K} A^{o p}$ é um anel simples e observe que $\phi(1 \otimes 1)=I d$ e que $\phi\left(\left(a \otimes_{K} b\right) \cdot\left(a^{\prime} \otimes_{K} b^{\prime}\right)\right)=$ $\phi\left(a \otimes_{K} b\right) \circ \phi\left(a^{\prime} \otimes_{K} b^{\prime}\right)$. Logo $\phi$ é um morfismo de $K$-álgebras. Assim Ker $\phi$ é um ideal bilaterar de $A \otimes_{K} A^{o p} \operatorname{logo} \operatorname{Ker} \phi=0$ e portanto $\phi$ é injetora.

E note que $\operatorname{dim} A \otimes_{K} A^{o p}=(\operatorname{dim} A)^{2}$ e como $\operatorname{End}_{K-m o d}(A)$ é isomorfo à álgebra das matrizes temos que $\operatorname{dim} \operatorname{End}_{K-\text { mod }}(A)=(\operatorname{dim} A)^{2} \operatorname{logo}$ pelo teorema do núcleo e imagem temos que $\operatorname{dim} \operatorname{End}_{K-\text { mod }}(A)=\operatorname{dim} A \otimes_{K} A^{o p}$ implica que $\phi$ é sobrejetiva.

$(\Leftarrow)$ Observe que pelo lema $Z(A) \otimes_{K} Z\left(A^{o p}\right)=Z\left(A \otimes_{K} A^{o p}\right)=Z\left(\operatorname{End}_{K-\text { mod }}(A)\right)=K$, segue que $Z(A)=K$. Por outro lado temos que $\operatorname{End}_{K-\bmod }(A)$ é simples, $\operatorname{logo} A$ também é simples, pois para cada ideal bilateral $I \subset A$ podemos conseguir um ideal bilateral $I \otimes_{K} A^{o p}$ de $A \otimes_{K} A^{o p}$ (observe que $A^{o p}$ é livre logo fielmente plano sobre $K)$.

Corolário 1.10. Seja $K$ um corpo e $\Omega$ uma extensão algebricamente fechada de $K$. Então são equivalentes:

1. A é um anel simples com centro $K$.

2. Existe um isomorfismo $A \cong M_{n}(D)$ onde $D$ é uma álgebra de divisão com centro $K$.

3. $A_{\Omega}$ é isomorfo a $M_{d}(\Omega)$ para algum $d$.

4. O mapa canônico $\phi: A \otimes_{K} A^{o p} \rightarrow \operatorname{End}_{K-m o d}(A)$ é um isomorfismo.

Demonstração. Segue diretamente dos teoremas anteriores.

Observe que para uma álgebra central simples $A$ temos

$$
\operatorname{dim}_{K} A=\operatorname{dim}_{\Omega} A \otimes \Omega=\operatorname{dim}_{\Omega} M_{n}(\Omega)=n^{2}
$$

de modo que $\operatorname{dim}_{K} A$ é sempre um quadrado perfeito.

Definição 1.11. O grau de uma álgebra central simples $A$ é definido como $\sqrt{\operatorname{dim}_{K} A}$. 


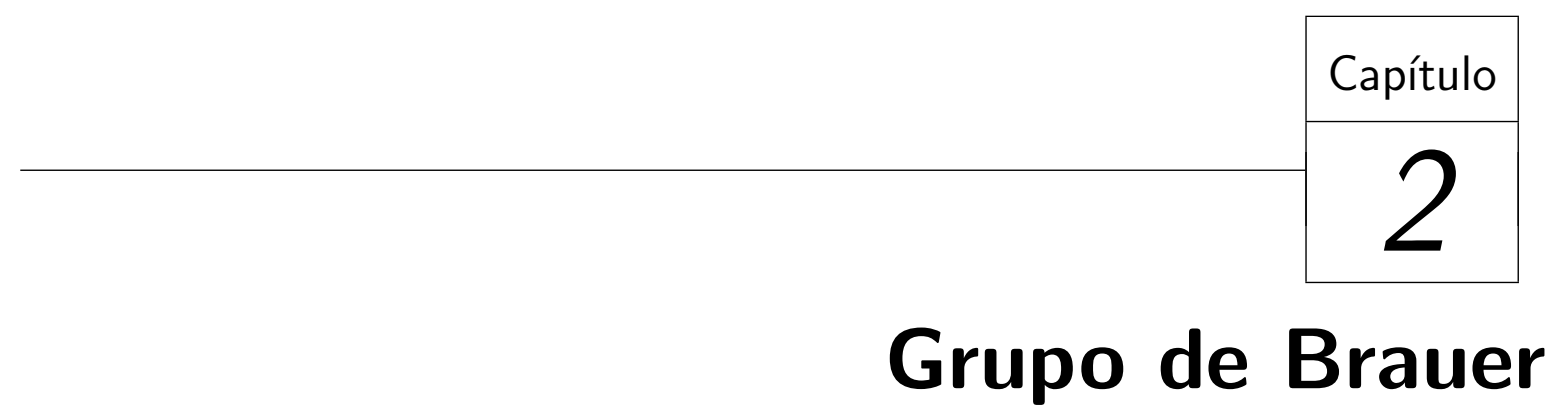

Grupo de Brauer

Como vimos no capítulo anterior todas as álgebras centrais simples ficam isomorfas ao anel de matrizes quando tensorizados por um corpo algebricamente fechado. Ou seja, álgebras centras simples são formas torcidas de matrizes. Neste capítulo veremos como classificar essas formas torcidas via um grupo de cohomologia. Em seguida definiremos o Grupo de Brauer e veremos como interpretá-lo como um grupo de cohomologia.

\subsection{Descenso de Galois}

Definição 2.1. Seja $V$ um espaço vetorial. Um tensor $\Phi$ do tipo $(p, q), p, q \geq 0$ inteiros, é um elemento do do produto tensorial $V^{\otimes p} \otimes\left(V^{*}\right)^{\otimes q}$.

Seja $V$ um espaço vetorial equipado com um tensor $\Phi$ do tipo $(p, q)$. Note que existe um isomorfismo natural.

$$
V^{\otimes p} \otimes\left(V^{*}\right)^{\otimes q} \cong \operatorname{Hom}_{K}\left(V^{\otimes q}, V^{\otimes p}\right)
$$

que segue da fórmula geral $\operatorname{Hom}_{K}(V, K) \otimes_{K} W \cong \operatorname{Hom}_{K}(V, W)$.

Exemplo 2.2. Seja A uma álgebra central simples sobre K. Podemos interpretar $A$ como um espaço vetorial sobre $K$ equipado com um tensor

$$
\Phi \in A \otimes\left(A^{*}\right)^{\otimes 2}=\operatorname{Hom}_{K}(A \otimes A, A)
$$

correspondente ao produto $A \otimes A \rightarrow A$ dado por $a \otimes b \mapsto a \cdot b$. 
Considere o par $(V, \Phi)$ de $K$-espaço vetorial equipado com um tensor do tipo $(p, q)$ fixado. Um $K$-isomorfismo entre dois objetos $(V, \Phi)$ e $(W, \Psi)$ é dado por um $K$ isomorfismo $f: V \rightarrow W$ de $K$-espaços vetoriais tal que $f^{\otimes p} \otimes\left(f^{*-1}\right)^{\otimes q}: V^{\otimes p} \otimes\left(V^{*}\right)^{\otimes q} \rightarrow$ $W^{\otimes p} \otimes\left(W^{*}\right)^{\otimes q}$ mapeia $\Phi$ em $\Psi$. Onde $f^{*}: W^{*} \rightarrow V^{*}$ é o $K$-isomorfismo induzido por $f$.

Agora fixe uma extensão Galois finita $L \mid K$ com grupo de Galois $G=\operatorname{Gal}(L \mid K)$. Denote por $V_{L}$ o $L$-espaço vetorial $V \otimes_{K} L$ e por $\Phi_{L}$ o tensor induzido em $V_{L}$ por $\Phi$. Assim associamos com $(V, \Phi)$ o $L$-objeto $\left(V_{L}, \Phi_{L}\right)$. Dizemos que $(V, \Phi)$ e $(W, \Psi)$ se tornam isomorfos sobre $L$ se existe um $L$-isomorfismo entre $\left(V_{L}, \Phi_{L}\right)$ e $\left(W_{L}, \Psi_{L}\right)$. Nesta situação $(W, \Psi)$ é também chamado de $L \mid K$-forma torcida de $(V, \Phi)$.

A teoria de Galois nos permite classificar classes $K$-isomorfismos de formas torcidas da seguinte maneira. Dado um $K$-automorfismo $\sigma: L \rightarrow L$, podemos considerar o $K$ automorfismo induzido Id $\otimes \sigma: V_{L} \rightarrow V_{L}$, que novamente denotaremos por $\sigma$. Cada mapa $L$-linear $f: V_{L} \rightarrow W_{L}$ induz um mapa $\sigma(f): V_{L} \rightarrow W_{L}$ definido por

$$
\sigma(f)=\sigma \circ f \circ \sigma^{-1}
$$

Se $f$ é um $L$-isomorfismo de $\left(V_{L}, \Phi_{L}\right)$ em $\left(W_{L}, \Psi_{L}\right)$, então $\sigma(f)$ também é. O mapa $f \rightarrow \sigma(f)$ preserva a composição de automorfismos, assim temos uma ação à esquerda de $G=\operatorname{Gal}(L \mid K)$ no grupo dos $L$-automorfismos de $\left(V_{L}, \Psi_{L}\right)$ que denotaremos aqui por $\operatorname{Aut}_{L}(\Psi)$. Mais que isso dado dois $K$-objetos $(V, \Phi)$ e $(W, \Psi)$ assim como um $L$-isomorfismo $g:\left(V_{L}, \Phi_{L}\right) \rightarrow\left(W_{L}, \Psi_{L}\right)$, obtemos uma mapa $G \rightarrow \operatorname{Aut}_{K}(\Phi)$ associando

$$
a_{\sigma}=g^{-1} \circ \sigma(g)
$$

para $\sigma \in G$. O mapa $a_{\sigma}$ satisfaz a seguinte relação fundamental

$$
a_{\sigma \tau}=a_{\sigma} \sigma\left(a_{\tau}\right), \quad \text { para todo } \quad \sigma, \tau \in G .
$$

De fato,

$$
\begin{aligned}
a_{\sigma \tau} & =g^{-1} \circ \sigma(\tau(g))=g^{-1} \circ \sigma(g) \circ \sigma\left(g^{-1}\right) \sigma(\tau(g)) \\
& =a_{\sigma} \circ \sigma\left(g^{-1}\right) \circ \sigma(\tau(g))=a_{\sigma} \circ \sigma\left(g^{-1} \circ \tau(g)\right) \\
& =a_{\sigma} \sigma\left(a_{\tau}\right) .
\end{aligned}
$$

Agora seja $h:\left(V_{L}, \Phi_{L}\right) \rightarrow\left(W_{L}, \Psi_{L}\right)$ um outro $L$-isomorfismo, definimos $b_{\sigma}:=$ $h^{-1} \sigma(h)$ para $\sigma \in G$. Assim $a_{\sigma}$ e $b_{\sigma}$ se relacionam por

$$
a_{\sigma}=c^{-1} b_{\sigma} \sigma(c)
$$

onde $c$ é o $L$-isomorfismo $h^{-1} \circ g$. 
Definição 2.3. Seja $G$ um grupo e A um outro grupo (não necessariamente comutativo) tal que $G$ age pela esquerda em $A$. Então um 1-cociclo de $G$ com valores em $A$ é um mapa $\sigma \mapsto a_{\sigma}$ de $G$ em A satisfazendo a relação 2.1.1 acima. Dois 1-cociclos $a_{\sigma}$ e $b_{\sigma}$ são chamados equivalentes ou cohomólogos se existe um $c \in A$ tal que a relação 2.1.2 é satisfeita.

Definição 2.4. Definiremos o primeiro grupo de cohomologia $H^{1}(G, A)$ de $G$ com valores em A como o conjunto quociente dos 1-cociclos pela relação de equivalência 2.1.2. Este é um conjunto pontuado, isto é, um conjunto equipado com um ponto distinto vindo do cociclo trivial $\sigma \mapsto 1$, onde 1 é o elemento identidade em A. Chamaremos este elemento de ponto base.

Em nossa situação concreta, vimos acima que a classe $\left[a_{\sigma}\right]$ em $H^{1}\left(G, \operatorname{Aut}_{L}(\Phi)\right)$ do 1cociclo $a_{\sigma}$ associado com o $L$-automorfismo $g:\left(V_{L}, \Phi_{L}\right) \rightarrow\left(W_{L}, \Psi_{L}\right)$ depende somente de $(W, \Psi)$ mas não depende de $g$. Com isso enunciaremos o seguinte teorema.

Teorema 2.5. Para um $K$-objeto $(V, \Phi)$ considere o conjunto pontuado $T F_{L}(V, \Psi)$ das $(L \mid K)$-formas torcidas de $(V, \Phi)$, com ponto base dado por $(V, \Phi)$. Então o mapa $(W, \Psi) \rightarrow\left[a_{\sigma}\right]$ definido acima produz uma bijeção preservando ponto base

$$
\theta: T F_{L}(V, \Psi) \leftrightarrow H^{1}\left(G, \operatorname{Aut}_{L}(\Phi)\right)
$$

Antes de provar este teorema precisaremos de um lema (ver [Ser79],p.151, proposition 3).

Lema 2.6 (Teorema 90 de Hilbert). Temos

$$
H^{1}\left(G, G L_{n}(L)\right)=\{1\}
$$

Demonstração. Seja $c \in M_{n}(L)$. Suponha que

$$
b=\sum_{\sigma \in G} a_{\sigma} \sigma(c)
$$

seja uma matriz invertível. Então $\tau(b)=a_{\tau}^{-1} b$. De fato, pela relação 2.1.1 temos $a_{\tau}^{-1} a_{\tau \sigma}=\tau\left(a_{\sigma}\right), \log \mathrm{O}$

$$
\tau(b)=\tau\left(\sum_{\sigma \in G} a_{\sigma} \sigma(c)\right)=\sum_{\sigma \in G} \tau\left(a_{\sigma}\right) \tau(\sigma(c))=a_{\tau}^{-1} \sum_{\sigma \in G} a_{\tau \sigma} \tau(\sigma(c))=a_{\tau}^{-1} b
$$

Vamos agora mostrar que é possível escolher $c$ como acima. Seja $x \in L^{n}$ e defina

$$
b(x)=\sum_{\sigma \in G} a_{\sigma} \sigma(x) \in L^{n}
$$


Afirmamos que os vetores $b(x)$ geram $L^{n}$ conforme $x$ percorre $L^{n}$. De fato, suponha por absurdo que exista um funcional linear não nulo $f$ tal que $f(b(x))=0 \forall x$. Se $\lambda \in L$, temos

$$
0=f(b(\lambda x))=\sum_{\sigma \in G} \sigma(\lambda) f\left(a_{\sigma} \sigma(x)\right)
$$

Pelo teorema de independência de caracteres de Dedekind [Lan02], Theorem 4.1, p.283, temos que $f\left(a_{\sigma} \sigma(x)\right)=0$ para todo $\sigma \in G$ e $x \in L^{n}$. Como $a_{\sigma}$ é invertível temos que $f$ é nulo. Absurdo.

Tome $x_{1}, \ldots, x_{n} \in L^{n}$ tais que $y_{i}=b\left(x_{i}\right)$ são linearmente independentes. Seja $c$ a matriz de mudança de base da base canônica para a base $x_{i}$. Temos que a matriz correspondente $b$ em 2.1.3 é a matriz de mudança de base da base canônica para $y_{i}$, logo $b$ é invertível como queríamos.

Prova do Teorema 2.5 ([Ser79], p.153, proposition 4). Vamos mostrar que $\theta$ é injetor. Sejam $\left(W_{1}, \Psi_{1}\right)$ e $\left(W_{2}, \Psi_{2}\right)$ duas formas torcidas com a mesma imagem por $\theta$, sejam

$$
f_{i}: V \otimes L \rightarrow W_{i} \otimes L
$$

os isomorfismos correspondentes. Podemos supor sem perda de generalidade que $f_{1}$ e $f_{2}$ geram o mesmo cociclo ou seja

$$
f_{1}^{-1} \sigma\left(f_{1}\right)=f_{2}^{-1} \sigma\left(f_{2}\right) \Longleftrightarrow \sigma\left(f_{2} f_{1}^{-1}\right)=f_{2} f_{1}^{-1}
$$

para todo $\sigma \in G$. Assim $f=f_{2} f_{1}^{-1}$ é um $K$-isomorfismo entre $\left(W_{1}, \Psi_{1}\right)$ e $\left(W_{2}, \Psi_{2}\right)$ $\log \theta$ é injetor.

Vamos agora provar que $\theta$ é sobrejetor. Seja $a: G \rightarrow \operatorname{Aut}_{L}(\Phi)$ um 1-cociclo. Como $\operatorname{Aut}_{L}(\Phi) \subset G L_{n}(L)$, pelo teorema 90 de Hilbert 2.6, temos que existe um $f \in G L_{n}(L)$ tal que

$$
a_{\sigma}=f^{-1} a_{\sigma}(f)
$$

. Defina $\Psi=f(\Phi)$. Este tensor está definido sobre $K$ : para todo $\sigma \in G$ temos

$$
\sigma(\Psi)=\sigma(f(\Phi))=\sigma(f) \sigma(\Phi)=\sigma(f) \Phi=f a_{\sigma}(\Phi)=f(\Phi)=\Psi
$$

(note que $\Phi$ está definido sobre $K$ e que $a_{\sigma}(\Phi)=\Phi$ pois $a_{\sigma} \in \operatorname{Aut}_{L}(\Phi)$ ). Assim $(V, \Psi)$ tem imagem dada pelo cociclo $a_{\sigma}$. 


\subsection{Grupo de Brauer}

Agora começaremos a classificar as álgebras centrais simples, primeiro relembraremos um fato bem conhecido do anel de matrizes.

Lema 2.7. Todo automorfismo sobre um corpo $K$ do anel das matrizes $M_{n}(K)$ é interno, isto é dado por $M \mapsto C M C^{-1}$ para alguma matriz invertível $C$.

Demonstração. Considere o ideal à esquerda minimal $I_{1}=\left[m_{i j}\right]$ tal que $m_{i j}=0$ se $j \neq 1$ e seja $\lambda \in \operatorname{Aut}\left(M_{n}(K)\right)$. Se necessário conjugamos $\lambda$ por uma matriz adequada e podemos assumir que $\lambda\left(I_{1}\right)=I_{1}$. Seja $e_{1}, \ldots, e_{n}$ a base canônica de $K^{n}$. Mapeando a matriz $M \in I_{1}$ em $M e_{1}$ induzimos um isomorfismo $I_{1} \cong K^{n}$ de $K$-espaços vetoriais, assim $\lambda$ induz um automorfismo de $K^{n}$, esse automorfismo é dado por uma matriz invertível $C$. Nós temos que para toda matriz $M \in M_{n}(K)$, o endomorfismo de $K^{n}$ definido na base canônica por $\lambda(M)$ é a matriz $C M C^{-1}$, e o lema segue.

Corolário 2.8. O grupo de automorfismos de $M_{n}(K)$ é o grupo projetivo linear $P G L_{n}(K)$.

Demonstração. Considere o morfismo

$$
\begin{aligned}
G L_{n}(K) & \rightarrow \operatorname{Aut}\left(M_{n}(K)\right) \\
C & \mapsto\left(M \mapsto C M C^{-1}\right)
\end{aligned}
$$

Pelo lema temos que o morfismo é sobrejetivo e seu núcleo é o centro do grupo $G L_{n}(K)$, isto é, o grupo das matrizes escalares.

Agora tome uma extensão Galois finita $L \mid K$, e seja $C S A_{L}(n)$ denota o conjunto das classes de $K$-isomorfismos das $K$-álgebras centrais simples de grau $n$ que cindem sobre $L$, que é um conjunto pontuado com ponto base a álgebra das matrizes $M_{n}(K)$.

Teorema 2.9. Existe uma bijeção preservando ponto base

$$
\theta: C S A_{L}(n) \longleftrightarrow H^{1}\left(G, P G L_{n}(L)\right)
$$

Demonstração. Vimos anteriormente que toda $K$-álgebra central simples de grau $n$ é precisamente uma forma torcida da álgebra de matrizes $M_{n}(K)$. Logo o resultado segue diretamente do teorema 2.5 .

Nosso próximo objetivo é classificar todas as $K$-álgebras de divisão que cindem sobre $L$ por meio de um conjunto de cohomologia. Este conjunto é munido de uma operação de produto, induzida pelo produto tensorial. 
Definição 2.10. Seja $K$ um corpo. Duas álgebras centrais simples $A$ e $B$ são ditas Brauer equivalentes se possuem a mesma álgebra de divisão subjacente, equivalentemente se

$$
A \otimes M_{n}(K) \cong B \otimes M_{m}(K)
$$

para algum $m, n$. É fácil ver que esta uma relação de equivalência no conjunto das classes de isomorfismo de álgebras centrais simples sobre $K$.

O grupo de Brauer de $K$, denotado por $B r(K)$, é o conjunto das classes de Brauer equivalência de álgebras centrais simples sobre $K$. Temos que $\operatorname{Br}(K)$ é um grupo abeliano com operação

$$
[A]+[B]=[A \otimes B]
$$

Pelo corolário 1.10, esta operação tem elemento neutro $[K]$ e inverso $-[A]=\left[A^{o p}\right]$.

Lema 2.11. Se $A$ e $B$ são $K$-álgebras centrais simples que cindem sobre $L$, então $A \otimes B$ também é uma $K$-álgebra central simples que cinde sobre $L$.

Demonstração. Lembramos que $\left(A \otimes_{K} L\right) \otimes_{L}\left(B \otimes_{K} L\right) \cong\left(A \otimes_{K} B\right) \otimes_{K} L$ e $M_{n}(L) \otimes_{L}$ $M_{m}(L) \cong M_{n m}(L)$ e pelo corolário 1.10 , temos o resultado

Definição 2.12. Seja $L \mid K$ uma extensão de corpos. O conjunto das classes de álgebras centrais simples sobre $K$ que cindem em $L$ formam um subgrupo de $B r(K)$, denotado por $\operatorname{Br}(L \mid K)$.

Seja $L \mid K$ uma extensão Galois com grupo de Galois $G$. Da seguência exata curta

$$
1 \longrightarrow L^{*} \longrightarrow G L_{n}(L) \longrightarrow P G L_{n}(L) \longrightarrow 1
$$

temos uma sequência exata de conjuntos pontuados ([Ser79], Proposition 2, p.125)

$$
\begin{aligned}
& 1 \longrightarrow H^{0}\left(G, L^{*}\right) \longrightarrow H^{0}\left(G, G L_{n}(L)\right) \longrightarrow H^{0}\left(G, P G L_{n}(L)\right) \\
& \longrightarrow H^{1}\left(G, L^{*}\right) \longrightarrow H^{1}\left(G, G L_{n}(L)\right) \longrightarrow H^{1}\left(G, P G L_{n}(L)\right) \\
& \stackrel{\Delta_{n}}{\longrightarrow} H^{2}\left(G, L^{*}\right)
\end{aligned}
$$

Pelo teorema 90 de Hilberto 2.6 temos que $\Delta_{n}$ é injetivo. Seja $\delta_{n}=\Delta_{n} \circ \theta: C S A_{L}(n) \rightarrow$ $H^{2}\left(G, L^{*}\right)$. Podemos verificar que dados $A \in C S A_{L}(n)$ e $B \in C S A_{L}(m)$ temos

$$
\delta_{n m}(A \otimes B)=\delta_{n}(A)+\delta_{m}(B)
$$

Além disso $\delta_{n}(A)=0 \Longleftrightarrow A=M_{n}(K)$.

Assim os mapas $\delta_{n}$ definem um homomorfismo injetivo

$$
\delta: \operatorname{Br}(L \mid K) \rightarrow H^{2}\left(G, L^{*}\right)
$$

Podemos mostrar ([Ser79], Proposition 9, p.158) que esse mapa também é sobrejetor, logo temos um isomorfismo entre $\operatorname{Br}(L \mid K)$ e $H^{2}\left(G, L^{*}\right)$. 


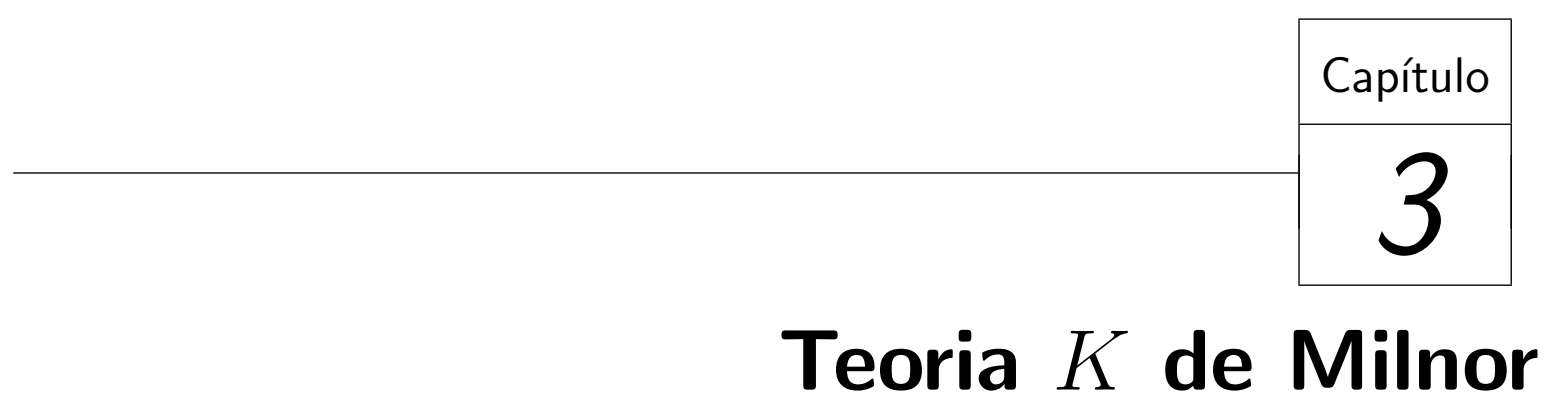

Neste capítulo faremos uma pequena revisão da teoria $K$ de Milnor. As referências para resultados deste capítulo são [GS06], capítulo 7, p.183 e [FV02], capítulo 9, p.233.

\subsection{Definições e Propriedades Básicas}

Seja $F$ um corpo. O anel graduado de Milnor

$$
K(F)=\bigoplus_{n \geq 0} K_{n} F
$$

de $F$ é o quociente da álgebra tensorial sobre $\mathbb{Z}$ do grupo multiplicativo $F^{*}$

$$
T\left(F^{*}\right)=\bigoplus_{n \geq 0}\left(F^{*}\right)^{\otimes n}
$$

pelo ideal gerado pelos tensores da forma $a_{1} \otimes a_{2} \otimes \ldots \otimes a_{n} \operatorname{com} a_{i}+a_{j}=1$, para $0 \leq i<j \leq n$. A classe do tensor $a_{1} \otimes a_{2} \otimes \ldots \otimes a_{n}$ em $K_{n}(F)$ é denotada por $\left\{a_{1}, a_{2}, \ldots, a_{n}\right\}$ e é chamado de símbolo.

Assim temos que $K_{0}(F)=\mathbb{Z}, K_{1}(F)=F^{*}$ e $K_{2}(F)$ é gerado pelos símbolos $\{a, b\}$, $a, b \in F^{*}$, com as seguintes relações:

$$
\begin{gathered}
\left\{a a^{\prime}, b\right\}=\{a, b\}+\left\{a^{\prime}, b\right\} \\
\left\{a, b b^{\prime}\right\}=\{a, b\}+\left\{a, b^{\prime}\right\} \\
\{a, b\}=0, \text { se } a+b=1
\end{gathered}
$$


Lema 3.1 (Propriedades Básicas de $K_{2}(F)$ ). Temos as seguintes identidades:

1. $\{1, a\}=\{a, 1\}=0 \forall a \in F^{*}$.

2. $\left\{\frac{1}{a}, b\right\}=-\{a, b\}$.

3. $\{a,-a\}=0 \forall a \neq 0$.

4. $\{a, b\}=-\{b, a\}$.

Demonstração. $\quad$ 1. $\{1, a\}=\{1 \cdot 1, a\}=\{1, a\}+\{1, a\} \Rightarrow\{1, a\}=0$

2. $0=\left\{\frac{a}{a}, b\right\}=\left\{\frac{1}{a}, b\right\}+\{a, b\}$.

3. O resultado é valido para $a=1$ pelo primeiro item. Para $a \neq 1$ note que $\{a,-a\}+\left\{a,-(1-a) a^{-1}\right\}=\{a, 1-a\}=0 \operatorname{logo}\{a,-a\}=-\left\{a,-(1-a) a^{-1}\right\}=$ $\left\{a^{-1}, 1-a^{-1}\right\}=0$.

4. $0=\{a b,-b a\}=\{a,-a\}+\{a, b\}+\{b, a\}+\{b,-b\}=\{a, b\}+\{b, a\}$.

Um homomorfismo de $\operatorname{corpos} \pi: F \rightarrow E$ induz um homomorfismo de anéis $K_{n}(F) \rightarrow K_{n}(E)$ dado por

$$
\left\{a_{1}, \ldots, a_{n}\right\} \mapsto\left\{\pi\left(a_{1}\right), \ldots, \pi\left(a_{n}\right)\right\}
$$

fazendo $K_{n}$ um funtor da categoria de corpos para a categoria dos grupos abelianos graduados.

\subsection{Morfismos Residual e de Especialização}

A proposição a seguir define os chamados mapas de especialização e residual; a demonstração pode ser encontrada em [FV02], §2, capítulo 9, p.226.

Proposição 3.2. Seja L um corpo com valorização discreta $v$, seja $A_{v}$ o anel de valorização e F o seu corpo residual. Seja $\pi$ um parâmetro local $(v(\pi)=1)$.

1. Para cada $n \geq 1$ existe um único homomorfismo (homomorfismo residual)

$$
\partial: K_{n}(L) \rightarrow K_{n-1}(F)
$$

satisfazendo

$$
\partial\left(\left\{\pi, u_{2}, \ldots, u_{n}\right\}\right)=\left\{\overline{u_{2}}, \ldots, \overline{u_{n}}\right\}
$$

para toda $(n-1)$-upla de unidades de $A_{v}$, onde $\overline{u_{i}}$ denota a imagem de $u_{i}$ em $F$. 
2. Existe um único homomorfismo de especialização, dado da seguinte forma

$$
s_{\pi}\left(\left(\pi^{i_{1}} u_{1}, \ldots, \pi^{i_{n}} u_{n}\right)\right)=\left\{\overline{u_{1}}, \ldots, \overline{u_{n}}\right\}
$$

Só utilizaremos a proposição acima para o caso $n=2$, para qual temos a seguinte descrição explicita:

Definição 3.3. Seja L um corpo com valorização discreta v e corpo residual F. Chamaremos de morfismo residual o seguinte morfismo

$$
\partial: K_{2} L \rightarrow K_{1} F
$$

definido por

$$
\partial(\{a, b\})=(-1)^{v(a) \cdot v(b)} \overline{\left(\frac{a^{v(b)}}{b^{v(a)}}\right)}
$$

Exemplo 3.4. Seja $F$ um corpo e seja $L=K(t)$. Seja v a valorização de $L$ definida pelo polinômio irredutível t. Diretamente das definições acima temos que

$$
s_{t}\left(u_{L}\right)=u \text { para todo } u \in K_{2} F
$$

Em particular $K_{2} F \rightarrow K_{2} L$ é injetor.

\subsection{Norma}

Para esta seção referenciamos [GS06] seção 7.3, p.195.

Teorema 3.5. Seja $E \mid F$ uma extensão finita. Para todo $n \neq 0$ existem mapas norma $N_{E \mid F}: K_{n} E \rightarrow K_{n} F$ com as seguintes propriedades:

1. O mapa $N_{E \mid F}: K_{0} E \rightarrow K_{0} F$ é a multiplicação por $[E: F]$.

2. O mapa $N_{E \mid F}: K_{1} E \rightarrow K_{1} F$ é a norma do corpo $N_{E \mid F}: E^{*} \rightarrow F^{*}$.

3. Dados $a \in K_{n}(F)$ e $b \in K_{m}(E)$, temos

$$
N_{E \mid F}(\{a, b\})=\left\{a, N_{E \mid F}(b)\right\}
$$

4. Dada uma torre de corpos $E^{\prime}|E| F$ então

$$
N_{E^{\prime} \mid F}=N_{E \mid F} \circ N_{E^{\prime} \mid E}
$$

Em particular, observe que $s \in K_{n} F$, então $N_{E \mid F}\left(s_{E}\right)=[E: F] \cdot s$. 



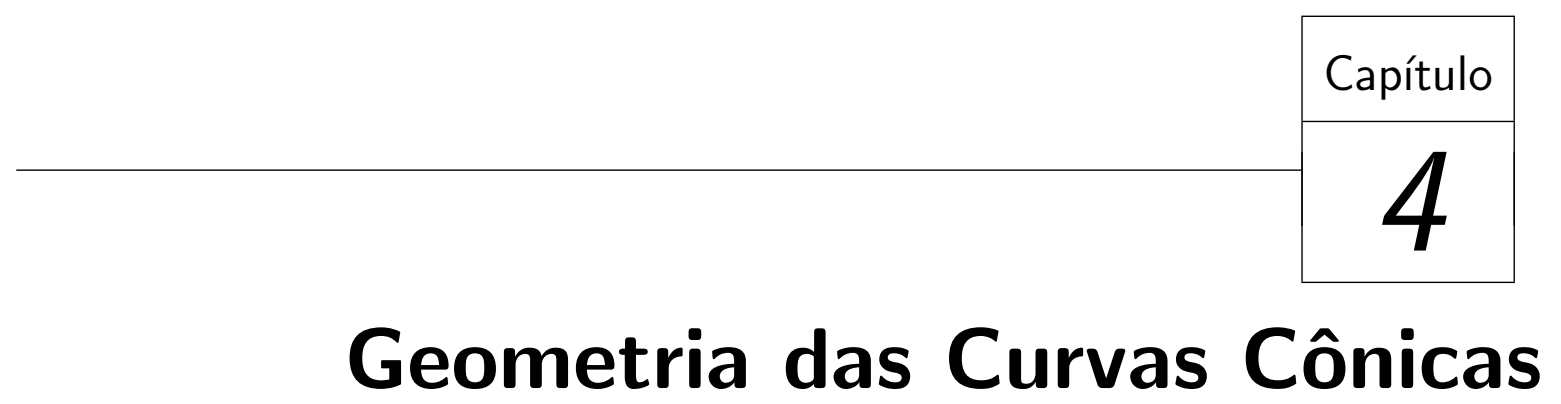

Estudaremos neste capítulo álgebras de quatérnios e sua relação com cônicas projetivas planas.

\subsection{Quatérnios}

Definição 4.1. Seja F um corpo (de característica arbitrária). Uma F-álgebra de quatérnios é uma F-álgebra central simples de grau 2 sobre $F$, ou seja, de dimensão 4 sobre $F$.

Exemplo 4.2. Seja $L / F$ uma extensão de corpos galoisiana quadrática e seja $b \in F^{\times}$. Definimos a álgebra de quatérnios $(L / F, b)$, como o espaço vetorial $L \oplus L v$ onde $v$ é um símbolo, com a seguinte regra de multiplicação, $v^{2}=b$ e $x v=\sigma(x) v$, onde $\sigma$ é $o$ gerador do grupo de Galois $\operatorname{Gal}(L / F)$ e $x \in L$.

Proposição 4.3 ([EKM08], Proposition 98.9, p.390). Toda F-álgebra de quatérnios é isomorfa a $(L / F, b)$ para alguma extensão quadrática $L / F$ e algum $b \in F^{\times}$.

Se $\operatorname{char}(\mathrm{F}) \neq 2$, temos que $L=F(\sqrt{a})$, para algum $a \in F^{\times}$e escreveremos $(a, b)_{F}$ no lugar de $(L / F, b)$.

Proposição 4.4 ([GS06], proposition 1.1.7, p.3). Seja $(a, b)$ uma álgebra de quatérnios sobre F. São equivalentes:

1. A álgebra $(a, b)$ cinde. 
2. A álgebra $(a, b)$ não é uma álgebra de divisão.

3. O mapa norma $N:(a, b) \rightarrow F$ possui um zero não trivial.

4. O elemento b é uma norma da extensão $F(\sqrt{a}) \mid F$.

\subsection{Norma e Traço Reduzidos}

Definição 4.5. Seja $Q=(L / F, b)$ uma álgebra de quatérnios, temos as seguintes aplicações

1. Involução Canônica

$$
\begin{aligned}
-: & \longrightarrow Q \\
& a \longrightarrow \bar{a} \\
& (x+y v) \longrightarrow \sigma(x)-y v, \text { para o gerador } \sigma \in \operatorname{Gal}(L / F)
\end{aligned}
$$

2. Traço Reduzido é um mapa linear dado por

$$
\begin{aligned}
& \text { Trd }: Q \longrightarrow F \\
& a \longrightarrow a+\bar{a}
\end{aligned}
$$

3. Norma Reduzida é definida por

$$
\begin{aligned}
\text { Nrd }: Q \longrightarrow F \\
a \longrightarrow a \cdot \bar{a}
\end{aligned}
$$

Proposição 4.6. Todo elemento $a \in Q=(L / F, b)$ satisfaz a equação

$$
a^{2}-\operatorname{Trd}(a) \cdot a+N r d(a)=0
$$

Demonstração. Seja $a=x+y v$, observe que $a \bar{a}=\bar{a} a$ e assim

$$
a^{2}-(a+\bar{a}) a+a \bar{a}=-\bar{a} a+a \bar{a}=0
$$




\subsection{Cônicas e Quatérnios}

Aqui estabeleceremos uma relação entre cônicas projetivas e álgebras de quatérnios sobre um corpo $F$ de característica diferente de 2 .

Seja $Q=(L \mid F, c)$ uma álgebra de quatérnios. Defina

$$
V=\operatorname{ker}(\operatorname{Tr} d)=\{a \in Q \mid \bar{a}=-a\}
$$

Como $L=F(\sqrt{b})$ podemos escrever qualquer elemento $a \in Q$ da seguinte forma:

$$
a=(x+y i)+(z+w i) v=x+y i+z v+w i v \text { onde } x, y, z, w \in F \text { e } i^{2}=b
$$

Assim temos que $\bar{a}=x-y i-z v-w i v$ e portanto $a=-\bar{a}$ se e somente se $x=0$. Logo $V=[i, v, i v]$.

Considere a forma bilinear em $Q$ dada por:

$$
\langle a, b\rangle \mapsto \operatorname{Trd}(a b)
$$

Esta forma bilinear é não degenerada: fixado $a$, se $\langle a, b\rangle=0$, para todo $b \in Q$, tomando $b=1, i, v, i v$, obtemos um sistema linear homogêneo nas coordenadas de $a$, e resolvendo-o obtemos $a=0$. Temos ainda que $V^{\perp}=[1]$ com respeito à forma bilinear não degenerada.

Da proposição anterior temos que $a^{2}=-N r d(a) \in F$ para todo $a \in V$ e, além disso, $\langle a, a\rangle=a^{2}+\bar{a}^{2}=a^{2}+(-a)^{2}=2 a^{2}$. Assim, como $\operatorname{char}(F) \neq 2, q(x)=x^{2}$ é uma forma quadrática em $V$ e a equação $q(x)=0$ define uma cônica projetiva suave $C$ no plano projetivo $\mathbb{P}(V)$.

Proposição 4.7. As seguintes condições são equivalentes

1. Q cinde

2. $C$ é isomorfo a $\mathbb{P}^{1}(F)$

3. $C$ tem ponto F-racional.

Demonstração. $\quad$ 1. $(1 \Rightarrow 2)$ : Como $Q$ é isomorfa à álgebra de matrizes $M_{2}(F), V$ é o espaço das matizes de traço nulo e $C$ é dada pela equação $t_{0}^{2}+t_{1} t_{2}=0$. O morfismo $C \rightarrow \mathbb{P}(V)$, dado por $\left[t_{0}: t_{1}: t_{2}\right] \mapsto\left[t_{0}: t_{1}\right]=\left[-t_{2}: t_{0}\right]$ é um isomorfismo.

2. $(2 \Rightarrow 3)$ : Óbvio. 
3. $(3 \Rightarrow 1)$ : Temos um elemento não nulo $x \in Q$ tal que $x^{2}=0$ logo $Q$ não é uma álgebra de divisão, assim $Q$ é isomorfo a uma álgebra de matrizes.

Exemplo 4.8. Seja char $F \neq 2$ e seja $1, i, j, k$ uma base de $Q$ com, $i j=-j i=k$ e $a=i^{2}, b=j^{2}, a, b \in F^{\times}$. Assim $V=F i \oplus F j \oplus F k$ e $C$ é dada pela equação $a x^{2}+b y^{2}-a b z^{2}=0$.

Demonstração. Seja $h \in V$ temos que $q(h)=0 \Rightarrow a x^{2}+b y^{2}-a b z^{2}+x y i j+x y j i+$ $z x k i+x z i k+y z j k+z y k j=0 \Rightarrow a x^{2}+b y^{2}-a b z^{2}=0$.

O seguinte lema será utilizado na demonstração do teorema 5.4.

Lema 4.9. Sejam $(a, b)_{F} e(c, d)_{F}$ duas álgebras de quatérnios sobre um corpo $F$ de característica não 2 isomorfas. Então existe um $e \in F^{\times}$satisfazendo

$$
(a, b)_{F} \cong(a, e)_{F} \cong(c, e)_{F} \cong(c, d)_{F}
$$

Demonstração. Note que se temos $x, y \in V$ em uma álgebra de quatérnios $Q$, com $x$ e $y$ ortogonais com respeito à forma bilinear traço reduzido, i.e, $\operatorname{Trd}(x y)=0$, então $Q \cong\left(x^{2}, y^{2}\right)$. Basta tomar o morfismo $i \mapsto x$ e $v \mapsto y$, observando que como $\operatorname{Tr} d(x y)=$ $0 \Longleftrightarrow x y=-y x$ as relações da álgebra são mantidas.

Seja $Q=(a, b)$. Podemos assumir que existem $x, y$ satisfazendo $x^{2}=a$ e $y^{2}=c$. Escolha um $z \in V$ ortogonal a $x$ e $y$ e escolha $e=z^{2}$, logo temos $Q \cong(a, e) \cong(c, e)$. 


\section{$\frac{5}{5}$ \\ O Teorema de Merkurjev-Suslin}

Neste capítulo, provaremos o teorema de Merkurjev-Suslin: para todo corpo $F$ com $\operatorname{char}(F) \neq 2$, temos que o símbolo de Galois

$$
h_{F}: \frac{K_{2} F}{2 K_{2} F} \rightarrow_{2} \operatorname{Br}(F)
$$

é um isomorfismo. Seguiremos o artigo [Mer06] de A. Merkurjev, onde ele dá uma prova que simplifica a prova original do artigo [MS82].

\subsection{Símbolo de Galois}

Seja $F$ um corpo de característica diferente de 2. Para cada $a, b \in F^{\times}$a classe da álgebra de quatérnios $(a, b)_{F}$ no Grupo de Brauer $\operatorname{Br}(F)$ tem ordem 2:

$$
(a, b) \otimes(a, b) \cong(a, b) \otimes(a, b)^{o p} \cong M_{4}(F)
$$

Mais que isso, a álgebra $(a, b)_{F}$ cinde se $a+b=1$, pois neste caso $b=1-a=$ $N_{F(\sqrt{a}) \mid F}(1+\sqrt{a})$ (ver proposição 4.4$)$. Note ainda que a classe de $(a, b)_{F}$ é bilinear com respeito a $a$ e $b$. Assim temos um morfismo bem definido

$$
h_{F}: K_{2} F / 2 K_{2} F \rightarrow_{2} \operatorname{Br}(F)
$$

levando $\{a, b\}+2 K_{2} F$ para a classe da álgebra de quatérnios $(a, b)_{F}$.

Seja $L$ um corpo com valorização discreta $v$ e corpo residual $F$. Lembre-se (definição 3.3) que temos um morfismo residual

$$
\partial: K_{2} L \rightarrow K_{1} F
$$


definido por $\partial(\{a, b\})=(-1)^{v(a) \cdot v(b)}\left(\overline{\frac{a^{v(b)}}{b^{v(a)}}}\right)$. Se $C$ uma curva suave sobre um corpo $F$, para cada ponto fechado $x \in C$ temos também um morfismo residual

$$
\partial_{x}: K_{2} F(C) \rightarrow K_{1} F(x)=F(x)^{\times}
$$

induzido pela valorização discreta do anel local $O_{C, x}$.

Utilizaremos os seguintes resultados técnicos para os quais nos referiremos ao artigo [Mer06].

Teorema 5.1 ([Mer06], Theorem 4.1, p.7). Seja C uma curva cônica sobre o corpo F . A seguinte sequência é exata

$$
K_{2} F \rightarrow K_{2} F(C) \stackrel{\partial}{\rightarrow} \amalg_{x \in C} F(x)^{\times} \stackrel{N}{\rightarrow} F^{\times},
$$

onde $\partial=\amalg \partial_{x}$ e $N$ é dado pelo mapa norma $N_{F(x) / F}$.

Teorema 5.2 ([Mer06], Theorem 5.4, p.21). Seja L/F uma extensão quadrática Galois e seja $\sigma$ o gerador de $\operatorname{Gal}(L / F)$. Então a seguinte sequência é exata

$$
K_{2} L \stackrel{1-\sigma}{\rightarrow} K_{2} L \stackrel{N_{L / F}}{\rightarrow} K_{2} F
$$

Teorema 5.3 ([Mer06], Theorem 5.5, p.21). Seja $u \in K_{2} F$ um elemento tal que $2 u=0$. Então $u=\{-1, a\}$ para algum $a \in F^{\times}$.

\subsection{Demonstração do Teorema de Merkurjev-Suslin}

Teorema 5.4. (Teorema de Merkurjev-Suslin) Para cada corpo F de característica não 2,

$$
h_{F}: K_{2} F / 2 K_{2} F \rightarrow_{2} \operatorname{Br}(F)
$$

é um isomorfismo.

Injetividade de $h_{F}$. Suponha que $h_{F}\left(u+2 K_{2} F\right)=1$ para algum elemento $u \in K_{2} F$ e que $u$ seja a soma de $n$ símbolos. Provaremos por indução sobre $n$ que $u \in K_{2} F$.

Caso $n=1$.

Temos que $u=\{a, b\}, a, b \in F^{\times}$. Como $(a, b)_{F}$ é uma álgebra de quatérnios que cinde temos que $b=x^{2}-a y^{2}$ para algum $x, y \in F$ pela proposição 4.4. Vamos mostrar 
que $u=0$. De fato, se $x \neq 0$, note que

$$
\begin{aligned}
0 & =\left\{a\left(y x^{-1}\right)^{2}, 1-a\left(y x^{-1}\right)^{2}\right\} \\
& =\left\{a, \frac{x^{2}-a y^{2}}{x^{2}}\right\}+\left\{\left(\frac{y}{x}\right)^{2}, \frac{x^{2}-a y^{2}}{x^{2}}\right\} \\
& =\left\{a, x^{2}-a y^{2}\right\}+2\left(\left\{\frac{y}{x}, \frac{x^{2}-a y^{2}}{x^{2}}\right\}-\{a, x\}\right)
\end{aligned}
$$

Se $x=0$ temos que $u=\left\{a,-a y^{2}\right\}=\{a,-a\}+2\{a, y\}=2\{a, y\}$. Logo $\left\{a, x^{2}-a y^{2}\right\} \in$ $2 K_{2} F$.

Caso $n=2$.

Temos $u=\{a, b\}+\{c, d\}$. Por hipótese temos que $(a, b)_{F} \otimes(c, d)_{F}$ cinde, portanto $\left[(a, b)_{F}\right]+\left[(c, d)_{F}\right]=0 \Longleftrightarrow\left[(a, b)_{F}\right]=-\left[(c, d)_{F}\right]=\left[(c, d)_{F}\right]$ em $\operatorname{Br}(F)$, ou seja, $(a, b)_{F} \cong(c, d)_{F}$. Logo pelo lema 4.9 podemos assumir $a=c$ e assim $u=\{a, b d\}$. Pelo caso $n=1$ segue o resultado.

\section{Caso geral.}

Escreveremos $u=\{a, b\}+v$, com $a, b \in F^{\times}$e $v \in K_{2} F$ sendo uma soma de $n-1$ símbolos. Seja $C$ a cônica sobre $F$ correspondente à álgebra de quatérnios $Q=(a, b)_{F}$ e fixe $L=F(C)$. A cônica $C$ é dada pela equação

$$
a X^{2}+b Y^{2}-a b Z^{2}=0
$$

em coordenadas projetivas; tome $x=\frac{X}{Z}$ e $y=\frac{Y}{Z}$. Seja $p \in C$ o ponto de grau 2 dado por $Z=0$.

Inicialmente vamos mostrar que

$$
\{a, b\}=2 r \text { em } K_{2} L \text { para } r=\left\{x, \frac{y^{2}}{a}\right\}-\{b, y\}
$$

De fato

$$
\begin{aligned}
\frac{x^{2}}{b}+\frac{y^{2}}{a}=1 & \Rightarrow \frac{x^{2}}{b}=1-\frac{y^{2}}{a} \Rightarrow \\
0 & =\left\{\frac{x^{2}}{b}, \frac{y^{2}}{a}\right\} \\
& =2\left\{x, \frac{y^{2}}{a}\right\}-\left\{b, y^{2}\right\}+\{b, a\} \\
& =2\left\{x, \frac{y^{2}}{a}\right\}-2\{b, y\}-\{a, b\} \\
& \Rightarrow\{a, b\}=2\left(\left\{x, \frac{y^{2}}{a}\right\}-\{b, y\}\right)=2 r
\end{aligned}
$$




\section{Lema 5.5.}

$$
\partial_{q}(r)=\left\{\begin{aligned}
-1 & \text { se } q=p \\
1 & \text { caso contrário }
\end{aligned}\right.
$$

Provaremos este lema mais tarde. Como a álgebra de quatérnios $(a, b)_{F}$ cinde sobre $L$, temos que $h_{L}\left(v_{L}+2 K_{2} L\right)=0$. Por indução

$$
v_{L}=2 w
$$

para algum elemento $w \in K_{2} L$.

Agora, a partir de $w$, vamos construir um elemento $w^{\prime} \in K_{2} L$ tal que $\partial_{q}\left(w^{\prime}\right)=1$ para todo $q \in C$. Defina

$$
c_{q}=\partial_{q}(w)
$$

para cada ponto $q \in C$. Assim, como $v \in K_{2} F$, pelo teorema 5.1 temos que

$$
c_{q}^{2}=\partial_{q}(w)^{2}=\partial_{q}(2 w)=\partial_{q}\left(v_{L}\right) \stackrel{5.1}{=} 1 .
$$

Assim temos que

$$
c_{q}=(-1)^{n_{q}} \text { onde } n_{q}=1 \text { ou } 0
$$

Como o grau de todo ponto em $C$ é par temos que

$$
\sum_{q \in C} n_{q} \operatorname{deg}(q)=2 m
$$

para algum $m \in \mathbb{N}$. Como todo divisor de grau zero em $C$ é principal (por exemplo, pelo teorema de Riemann-Roch), existe uma função $f \in L^{\times}$tal que

$$
\operatorname{div}(f)=\sum n_{q} q-m p
$$

Defina

$$
w^{\prime}=w+\{-1, f\}+k r \in K_{2} L \text { onde } k=m+n_{p}
$$

Se $q \in C$ é um ponto diferente de $p$, nós temos

$$
\begin{aligned}
\partial_{q}\left(w^{\prime}\right) & =\partial_{q}(w) \cdot \partial_{q}(\{-1, f\}) \cdot \partial_{q}(r)^{k} \\
& \stackrel{5.5}{=}(-1)^{n_{q}} \cdot(-1)^{v_{q}(-1) v_{q}(f)}\left(\overline{\frac{(-1)^{v_{q}(f)}}{f^{v_{q}(-1)}}}\right) \cdot 1^{k} \\
& =(-1)^{2 n_{q}}=1
\end{aligned}
$$

Analogamente se $q=p$

$$
\partial_{p}\left(w^{\prime}\right)=\partial_{p}(w) \cdot(-1)^{m} \cdot(-1)^{k}=(-1)^{n_{p}+m+k}=(-1)^{2 k}=1,
$$


Logo nós temos que $\partial_{q}\left(w^{\prime}\right)=1$ para todo $q \in C$. Pelo teorema 5.1,

$$
w^{\prime}=s_{L} \text { para algum } s \in K_{2} F
$$

Assim, como $2\{-1, f\}=0$ e $\{a, b\}=2 r$, temos

$$
v_{L}=2 w=2 w^{\prime}-2 k r=2 s_{L}-\left\{a^{k}, b\right\}_{L}
$$

Defina $v^{\prime}=v-2 s+\left\{a^{k}, b\right\} \in K_{2} F$, assim por construção temos que $v_{L}^{\prime}=0$.

A cônica $C$ cinde sobre a extensão quadrática $E=F(\sqrt{a})$, ou seja, $C$ possui um ponto $E$-racional, assim $C \cong \mathbb{P}_{E}^{1}$ e, desta forma, a extensão $E(C) / E$ é puramente transcendente, assim $K_{2}(E) \rightarrow K_{2}(E(C))$ é injetor pelo exemplo 3.4.

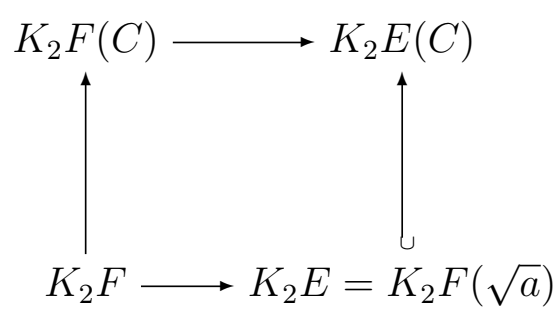

Como $v_{L}^{\prime}=0$ temos $v_{E(C)}^{\prime}=0, \operatorname{logo} v_{E}^{\prime}=0$ e assim $2 v^{\prime}=N_{E / F}\left(v_{E}^{\prime}\right)=0$. Pelo teorema 5.3, $v^{\prime}=\{-1, d\}$ para algum $d \in F^{\times}$. Assim o elemento $v$ módulo $2 K_{2} F$ é a soma de dois símbolos $\left\{a^{k}, b\right\}$ e $\{-1, d\}$. E isso se reduz ao caso $n=2$.

Para mostrar a sobrejetividade precisaremos da seguinte proposição.

Proposição 5.6. Seja $L \mid F$ uma extensão quadrática. Então a seguinte sequência é exata:

$$
\frac{K_{2} F}{2 K_{2} F} \rightarrow \frac{K_{2} L}{2 K_{2} L} \stackrel{N_{L \backslash F}}{\rightarrow} \frac{K_{2} F}{2 K_{2} F}
$$

Demonstração. Seja $u \in K_{2} L$ tal que $N_{L \mid F}(u)=2 v$ para algum $v \in K_{2} F$. Então $N_{L \mid F}\left(u-v_{L}\right)=2 v-2 v=0$ e pelo teorema $5.2, u-v_{L}=(1-\sigma) w$ para algum $w \in K_{2} L$. Assim

$$
u=v_{L}+(1-\sigma) w=\left(v+N_{L \mid F}(w)\right)_{L}-2 \sigma w
$$


Sobrejetividade de $h_{F}$. Vamos inicialmente provar um caso particular onde $F$ não possui extensão de grau ímpar. Seja $s \in_{2} B r F$. A prova é por indução no índice de $s$, ou seja o grau da álgebra de divisão na classe de $s$.

Seja $L \mid F$ uma extensão quadrática tal que o índice de $s_{L}$ é estritamente menor que o índice de $s$. Temos o diagrama comutativo ([GS06] Proposition 7.5.5, p.211)

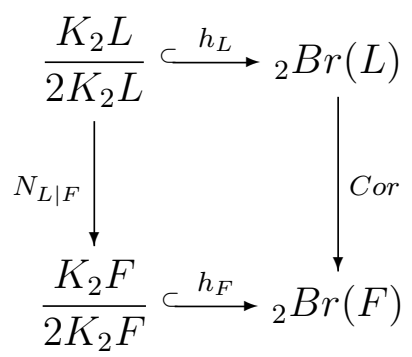

Seja $u \in K_{2} L$ tal que $h_{L}(u)=s_{L}$, que existe por hipótese de indução. Pelo diagrama acima temos

$$
h_{F}\left(N_{L \mid F}(u)\right)=\operatorname{Cor}\left(h_{L}(u)\right)=\operatorname{Cor}\left(s_{L}\right)=2 s=0
$$

Pela injetividade de $h_{F}$ temos que $N_{L \mid F}(u)=0$. Pela proposição anterior temos que $u \equiv v_{L}$ módulo $2 K_{2} F$ para algum $v \in K_{2} F$.

Note que $L$ cinde $s-h_{F}(v)$ pois

$$
h_{L}(u)=s_{L} \Longleftrightarrow h_{L}(u)-s_{L}=0 \Longleftrightarrow h_{L}\left(v_{L}\right)-s_{L}=0 \Longleftrightarrow\left(h_{F}(v)-s\right)_{L}=0
$$

Assim $s-h_{F}(v)$ é uma álgebra de quatérnios $(a, b)=h_{F}(\{a, b\}) \operatorname{logo} s=h_{F}(v+\{a, b\})$.

Para o caso geral, tome $F^{\prime}$ o compósito de todas as extensões de grau ímpar de $F$. Pelo caso especial acima, sabemos que existe $v \in K_{2} F^{\prime}$ tal que $h_{F^{\prime}}(v)=s_{F^{\prime}}$. Como $v$ é uma soma finita de símbolos existe uma subextensão finita $E$ de grau ímpar sobre $F$ tal que $v \in K_{2} E$ :

$$
F \stackrel{\text { ímpar }}{\subset} E \subset F^{\prime} \subset \bar{F}
$$

Assim, modulo $2 K_{2} F$ temos

$$
s=\operatorname{Cor}\left(s_{E}\right)=\operatorname{Cor}\left(h_{E}(v)\right) \stackrel{\text { diagrama }}{=} h_{F}\left(N_{E \mid F}(v)\right)
$$




\section{Referências Bibliográficas}

[EKM08] Richard Elman, Nikita Karpenko, and Alexander Merkurjev. The algebraic and geometric theory of quadratic forms, volume 56 of American Mathematical Society Colloquium Publications. American Mathematical Society, Providence, RI, 2008.

[FV02] I. B. Fesenko and S. V. Vostokov. Local fields and their extensions, volume 121 of Translations of Mathematical Monographs. American Mathematical Society, Providence, RI, second edition, 2002. With a foreword by I. R. Shafarevich.

[GS06] Philippe Gille and Tamás Szamuely. Central simple algebras and Galois cohomology, volume 101 of Cambridge Studies in Advanced Mathematics. Cambridge University Press, Cambridge, 2006.

[Lan02] Serge Lang. Algebra, volume 211 of Graduate Texts in Mathematics. SpringerVerlag, New York, third edition, 2002.

[Mer06] Alexander Merkurjev. On the norm residue homomorphism of degree two. In Proceedings of the St. Petersburg Mathematical Society. Vol. XII, volume 219 of Amer. Math. Soc. Transl. Ser. 2, pages 103-124, Providence, RI, 2006. Amer. Math. Soc.

[MS82] A. S. Merkur'ev and A. A. Suslin. K-cohomology of Severi-Brauer varieties and the norm residue homomorphism. Izv. Akad. Nauk SSSR Ser. Mat., 46(5):1011-1046, 1135-1136, 1982.

[Ser79] Jean-Pierre Serre. Local fields, volume 67 of Graduate Texts in Mathematics. Springer-Verlag, New York, 1979. Translated from the French by Marvin Jay Greenberg. 